\title{
Resultados preliminares del uso clínico de la electroquimioterapia en pacientes con cáncer de piel
}

Preliminary results of the clinical use of electrochemotherapy in patients with skin cancer

\author{
Wilson Galvis-Franco'ㅗ Felipe Jaramillo-Ayerbe², Juan Paulo Cardona 3 . \\ 1. Médico cirujano, residente de tercer año de Dermatología, Universidad de Caldas, Manizales, Colombia \\ 2. Médico dermatólogo, dermatopatólogo; director del posgrado de Dermatología, Universidad de Caldas, Manizales, Colombia \\ 3. Médico oncólogo; docente, Universidad de Caldas, Manizales, Colombia
}

\section{Resumen}

INTRODUCción. La electroquimioterapia es la estimulación eléctrica local de un tumor para tratarlo intralesionalmente con citotóxicos en dosis menores a las utilizadas tradicionalmente. Se describe el uso clínico de la electroquimioterapia en dos pacientes de Manizales con cáncer de piel.

OвJETIVo. Determinar el efecto de la electroquimioterapia en pacientes con cáncer de piel.

MATERIALES Y MÉTODos. Se trata de un estudio de casos en que se evaluó la respuesta clínica a la electroquimioterapia de dos pacientes con cáncer de piel, con valoraciones antes y después del tratamiento. Las valoraciones clínicas después del tratamiento fueron hechas por un dermatólogo independiente, no incluido en la investigación.

Resultados. Se utilizó la electroquimioterapia en dos pacientes. El primero, con un melanoma metastásico que presentaba en la piel del muslo derecho tres lesiones nodulares de $8 \mathrm{~mm}$ y un tumor de $3 \times 2 \mathrm{~cm}$, ulcerado y con sangrado constante. En el control a las cuatro semanas después del tratamiento, se evidenció ausencia de las lesiones tratadas y del sangrado activo, con remisión clínica completa (definida como desaparición completa del tumor). El segundo paciente era una mujer con un carcinoma escamocelular verrucoso de 6,5 x 3,5 $\mathrm{cm}$ en la vulva. En el control mensual se evidenció el tumor sin remisión clínica (sin cambios en la lesión).

ConClusión. La electroquimioterapia es una herramienta terapéutica novedosa y promisoria para el manejo coadyuvante o paliativo de pacientes con recidivas o cáncer de piel resistentes al tratamiento convencional. Se utilizó en los pacientes descritos con resultados alentadores en uno de ellos. Se requiere mayor experiencia para su recomendación y uso en el país.

PALABRAS CLAVE: cáncer de piel, electroquimioterapia, membrana celular, electroporación

\section{Summary}

INTRODUCTION: Electrochemotherapy is the electrostimulation of a tumor treated locally with cytotoxic drugs at lower doses than those traditionally used. We describe the clinical use of electrochemotherapy in Manizales in patients with skin cancer.

\section{Correspondencia: \\ Wilson Galvis}

Email:

wilsongalvisf@yahoo.com

Recibido: 16 de diciembre de 2013.

Aceptado: 12 de febrero de 2014.

No se reportan conflictos de interés. 
OBJEctive: To determine the effect of electrochemotherapy in patients with skin cancer.

MATERIALS AND METHODS: Case study, which evaluated the clinical response of two patients with skin cancer treated with electrochemotherapy with pre and post-treatment evaluations. Post-treatment clinical assessments were performed by an independent dermatologist.

RESULTS: Electrochemotherapy was performed in two patients; the first one with a metastatic melanoma presenting three $8 \mathrm{~mm}$ nodular lesions and a $30 \mathrm{x}$ $20 \mathrm{~mm}$ ulcerated and bleeding tumor in the right thigh; post-treatment monitoring at four weeks evidenced complete clinical response (defined as complete disappearance of the tumor). The second one was a patient with a $6.5 \times 3.5 \mathrm{~cm}$ vulvar verrucous squamous cell carcinoma; monthly monitoring of the tumor size showed no clinical response (no change in the lesion).

CoNCLUSION: Electrochemotherapy is a novel and promising therapeutic tool for adjuvant o palliative treatment of patients with recurrent or refractory skin cancer to conventional therapies. It was used in two patients with encouraging results in one of them. Larger experience is required for recommendation and use in our country.

KEY WORDS: Skin neoplasms, electrochemotherapy, cell membrane, electroporation.

\section{Introducción}

La incidencia del cáncer de piel ha aumentado a nivel mundial, principalmente en poblaciones de piel blanca donde es la enfermedad maligna más común. Se estima una incidencia anual de más de 1,5 millones de casos de cáncer de piel no melanoma en los Estados Unidos; es la forma más frecuente de cáncer en este país ${ }^{1}$.

A nivel nacional, este aumento se demostró con el estudio llevado a cabo en un centro de referencia de Colombia, donde el volumen total de pacientes con diagnóstico de cáncer de piel presentó un incremento de 6 casos por 1.000 pacientes con diagnóstico nuevo confirmado en el 2003 a 17 casos por 1.000 pacientes en el $2005^{2}$. A nivel local, en el registro de población de cáncer del quinquenio 2002-2006 en el departamento de Caldas, se reportaron 473 casos de cáncer de piel, después del cáncer de mama, cuello uterino y estómago ${ }^{3,4}$.

Un problema adicional al aumento de la incidencia de la enfermedad, es la consulta tardía al dermatólogo por parte de los pacientes con cáncer de piel, los cuales en algunos casos reciben atención con lesiones avanzadas que requieren tratamientos agresivos o paliativos ${ }^{5-7}$. En un estudio reciente se concluyó que el factor preponderante de la consulta tardía se relaciona con características y actitudes de los pacientes, aunque también su- gieren aspectos por mejorar en los diferentes niveles de atención en salud ${ }^{8}$.

El diagnóstico y tratamiento adecuados y oportunos del cáncer de piel son un reto diario para el especialista. Por esta razón, es importante la actualización en nuevas propuestas diagnósticas y terapéuticas que surgen como respuesta a la búsqueda constante de los investigadores; es así como aparece una nueva modalidad de tratamiento para tumores de piel: la electroquimioterapia, que consiste en la electroestimulación local de un tumor con aumento de la permeabilidad de la membrana celular, en forma transitoria y reversible, asociada a medicamentos citotóxicos en dosis menores a las utilizadas tradicionalmente ${ }^{9,10}$. La corriente eléctrica potencia la acción antineoplásica de la quimioterapia y, a su vez, minimiza los efectos colaterales que esta induce en el organismo. Los tumores presentan mayor conductividad eléctrica que los tejidos sanos adyacentes, son más electronegativos y la densidad de corriente eléctrica que circula por ellos corresponde a su parte activa, fenómeno explicado por su mayor contenido de agua. Las células cancerosas se despolarizan y sus cargas migran hacia las membranas, lo cual hace que su polaridad sea más negativa y, de esta manera, se comportan como mejores conductores de la corriente eléctrica ${ }^{11}$.

El Servicio de Dermatología de la Universidad de 


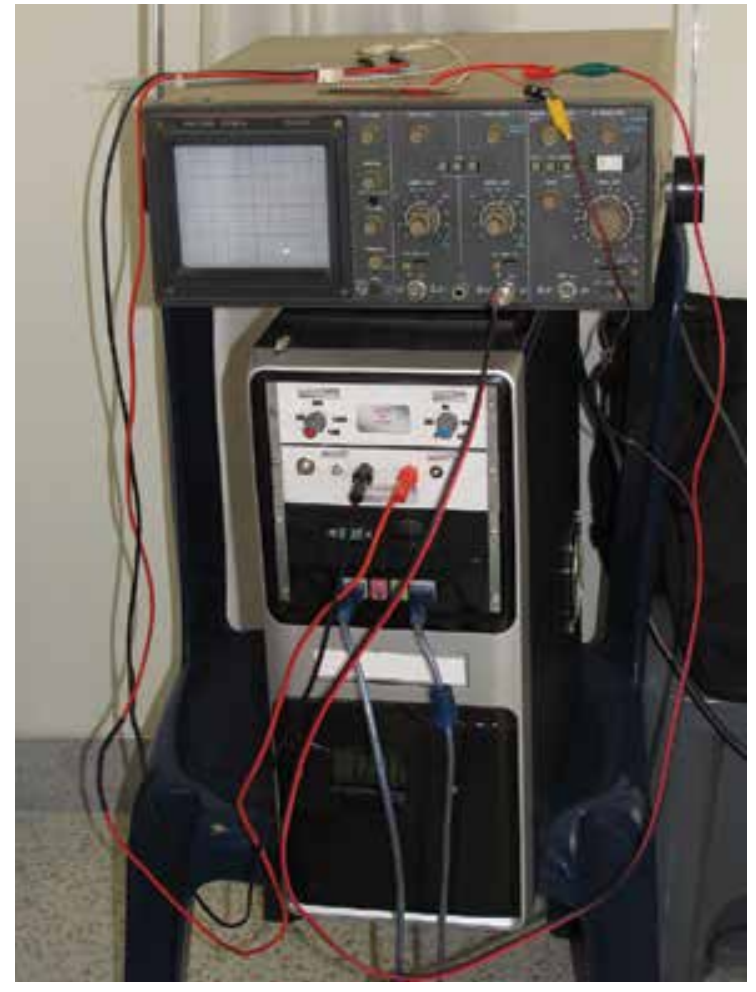

FIGURA 1. 'Electroporador', equipo médico utilizado para generar electro-permeabilización de la membrana celular.

Caldas, en asocio con ingenieros de la Universidad Nacional de Colombia, sede Manizales, mantiene abiertas líneas de investigación con talento humano idóneo. En concordancia con estas líneas investigativas, se propuso el desarrollo tecnológico local y el estudio clínico de la electroquimioterapia en pacientes con cáncer de piel, dado que se carece de experiencia sobre este tema en el ámbito nacional y local, y no se tienen datos en nuestra población de la respuesta a esta nueva alternativa de manejo.

El objetivo del estudio fue determinar el efecto de la electroquimioterapia en dos pacientes de Manizales con cáncer de piel y, el propósito, implementar su protocolo de manejo en el medio.

\section{Materiales y métodos}

Se desarrolló un estudio de casos, en el cual se evaluó la respuesta clínica a la electroquimioterapia de dos pacientes con cáncer de piel, con valoraciones antes y después del tratamiento. Las valoraciones clínicas después del tratamiento fueron hechas por un dermatólogo no incluido en la investigación para garantizar un estudio ciego.

Los criterios de inclusión utilizados fueron: pacientes con cáncer de piel de tipo melanoma y no melanoma, que no hubieran mejorado o no fueran candidatos a tratamientos convencionales, atendidos en el posgrado de Dermatología de la Universidad de Caldas y evaluados en la Clínica de Tumores de Piel y Mucosas, pacientes con diagnóstico de melanoma metastásico y en tránsito, y pacientes sin hipersensibilidad a los medicamentos utilizados en el estudio ni contraindicación clínica para el tratamiento. Se excluyeron los pacientes que no otorgaran consentimiento para practicar el tratamiento, con marcapasos o con hipersensibilidad a los medicamentos utilizados en el estudio.

En el período comprendido entre enero de 2012 y junio de 2013 dos pacientes cumplieron los criterios de inclusión. Se hizo un análisis descriptivo de los cambios en las características clínicas de las lesiones tratadas, entre ellas, la medición en centímetros de cada tumor.

Se elaboró e implementó el instrumento tecnológico ('electroporador') por parte del Departamento de Ingeniería de la Universidad Nacional de Colombia, sede Manizales (FIGURA 1), y el protocolo de uso y manejo de la electroquimioterapia en el Servicio de Dermatología y Oncología de la Universidad de Caldas.

Se obtuvo el aval del Comité de Bioética de la Facultad de Ciencias para la Salud y el consentimiento informado firmado por el paciente o su acudiente. El instrumento de recolección de datos fue diligenciado directamente por el investigador en la sesión inicial, y la evolución clínica del paciente después del tratamiento la evaluó un par (dermatólogo) no incluido en el estudio, el cual verificó a las cuatro semanas la respuesta clínica y la aparición de posibles efectos secundarios.

El protocolo utilizado para aplicar el tratamiento fue el establecido por el grupo de investigación con base en la literatura científica mundial. Se tomaron fotos y medidas de las lesiones antes y después del tratamiento. Se practicó una ecografía del tumor para establecer su volumen y aplicar la bleomicina intralesional, previa anestesia local perilesional con lidocaína al $1 \%$.

La bleomicina se dosificó según el volumen del tumor, así: 0,5 unidades para tumores de hasta $100 \mathrm{~mm}^{3} ; 0,75$ unidades para tumores de 100 a $150 \mathrm{~mm}^{3} ; 1$ unidad para tumores de 150 a $500 \mathrm{~mm}^{3} ; 1,5$ unidades para tumores de 500 a $1.000 \mathrm{~mm}^{3} ; 2$ unidades para tumores de $1.000 \mathrm{a}$ $2.000 \mathrm{~mm}^{3} ; 2,5$ unidades para tumores de 2.000 a 3.000 $\mathrm{mm}^{3} ; 3$ unidades para tumores de 3.000 a $4.000 \mathrm{~mm}^{3} ; 3,5$ unidades para tumores de 4.000 a $5.000 \mathrm{~mm}^{3}$, y 4 unidades para tumores mayores de $5.000 \mathrm{~mm}^{3}$.

La bleomicina se consigue en viales de 15 unidades para diluir en $10 \mathrm{ml}$ de solución salina normal y conservar a $-20^{\circ} \mathrm{C}$, ya que se deteriora después de 24 horas de reconstituida.

Diez minutos después de inyectar el medicamento, se 

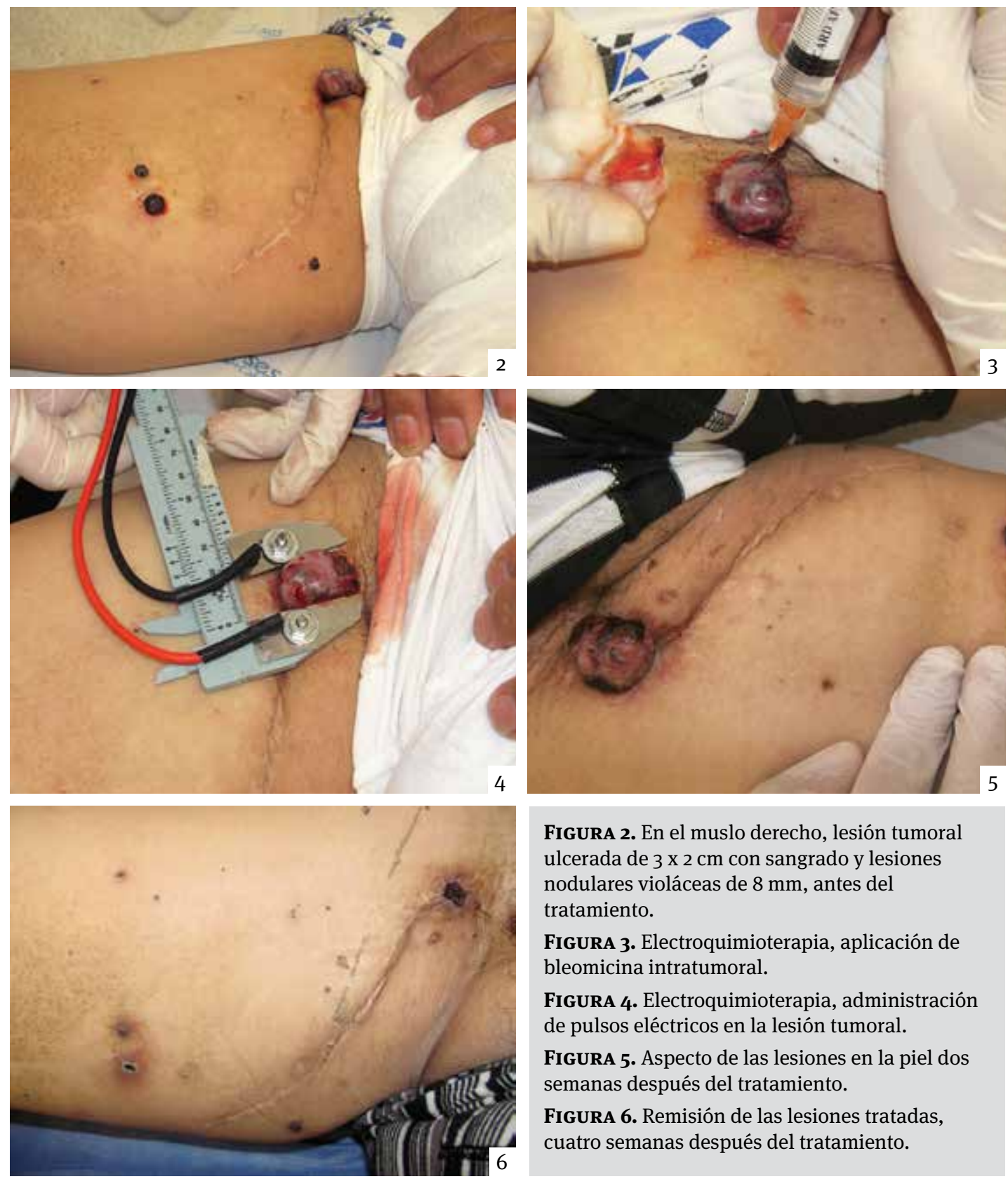

FIGURA 2. En el muslo derecho, lesión tumoral ulcerada de 3 x $2 \mathrm{~cm}$ con sangrado y lesiones nodulares violáceas de $8 \mathrm{~mm}$, antes del tratamiento.

FIGURA 3. Electroquimioterapia, aplicación de bleomicina intratumoral.

FIGURA 4. Electroquimioterapia, administración de pulsos eléctricos en la lesión tumoral.

FIGURA 5. Aspecto de las lesiones en la piel dos semanas después del tratamiento.

FIGURA 6. Remisión de las lesiones tratadas, cuatro semanas después del tratamiento.

administraron los pulsos eléctricos en cada lesión tumoral, a razón de 6 pulsos directos de corriente de 99 $\mu$ s en un campo estrecho de 1.300 voltios/cm, 1 pulso por segundo. La seguridad de la máquina se vigiló constantemente con un osciloscopio que demostraba la descarga exacta administrada al paciente, sin ningún riesgo para su vida.

Los pacientes fueron valorados durante el tratamiento e inmediatamente después y se hizo seguimiento telefónico los dos primeros días y, luego, valoración presencial a las cuatro semanas por un dermatólogo independiente, ajeno al estudio. Se observaron y anotaron los cambios de la lesión, dejando constancia con fotografías, además de valorar la respuesta clínica al final del seguimiento, y la aparición de efectos secundarios locales y sistémicos. La respuesta clínica se graduó como sigue; sin respuesta: sin cambios en la lesión; respuesta baja: reducción de menos del 50 \% de la lesión; respuesta intermedia: reducción de la lesión de 50 a 99\%; y, respuesta completa: cuando la lesión desaparece totalmente. 

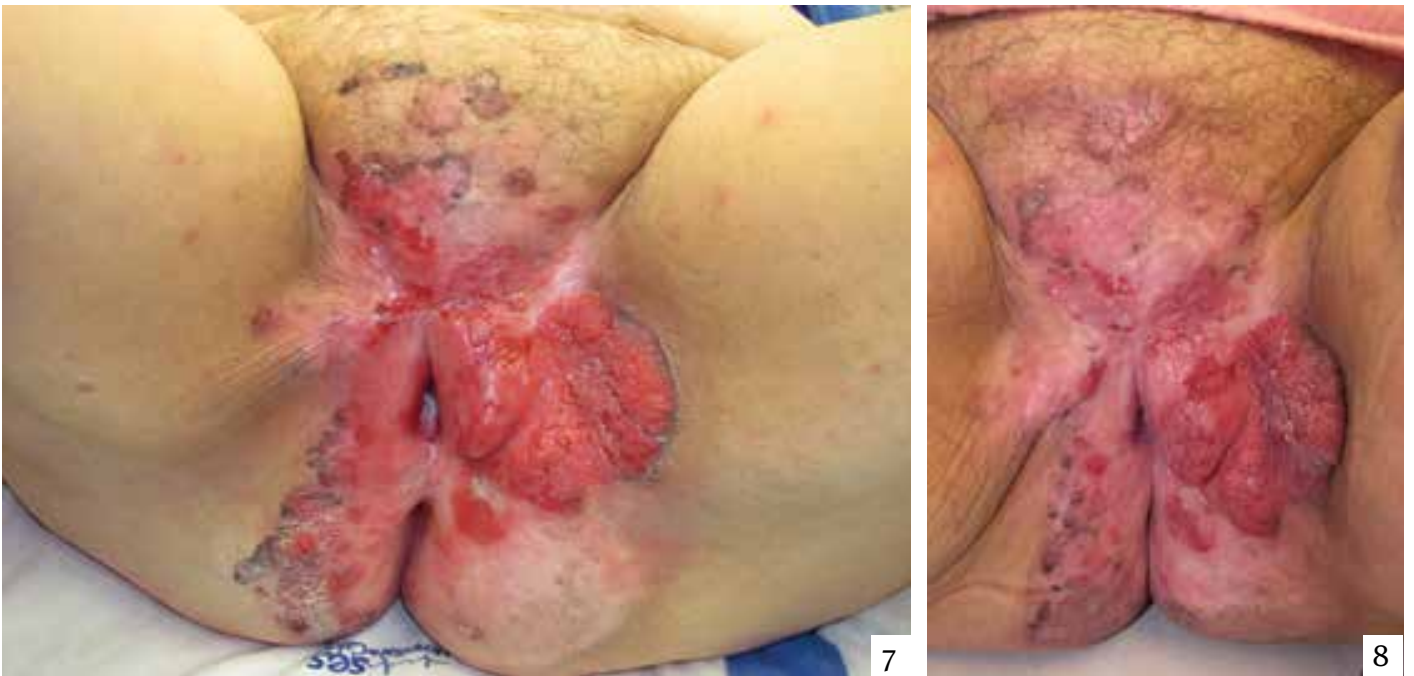

FIGURA 7. Tumor vulvo-inguinal izquierdo de $6,5 \times 3,5 \mathrm{~cm}$, antes del tratamiento.

FigurA 8. Aspecto de las lesiones en la piel, cuatro semanas después del tratamiento.

\section{Presentación de casos}

\section{Caso 1}

Se trata de un paciente de sexo masculino de 58 años de edad, con antecedentes de melanoma lentiginoso 'acral' (sic.) en el talón derecho, manejado inicialmente con cirugía oncológica y vaciamiento ganglionar inguinal derecho por aparición de adenopatía a este nivel, con reporte positivo para neoplasia en un ganglio de 14 estudiados. Se trató con interferón alfa en 40 sesiones.

Posteriormente, presentó lesiones metastásicas nodulares en la piel del muslo derecho y tumor sobre la cicatriz crural derecha con sangrado constante.

El caso fue analizado en el Comité de Tumores de Piel y Mucosas de la Universidad de Caldas y se decidió aplicar electroquimioterapia con bleomicina intratumoral.

En el examen físico se evidenciaban en el muslo derecho, lesiones nodulares violáceas de $8 \mathrm{~mm}$ de diámetro y una lesión tumoral ulcerada de $3 \times 2 \mathrm{~cm}$ con sangrado constante (FIGURA 2). En la ecografía del tumor se reportó un volumen de $1,5 \mathrm{~cm}^{3}$.

El tratamiento se administró según el protocolo mencionado anteriormente, previa anestesia local con lidocaína sin epinefrina. Se aplicaron 2 unidades de la dilución intratumoral en el tumor crural derecho y 0,5 unidades en los nódulos más pequeños del muslo (FIGURA 3). Diez minutos después de inyectar el medicamento, se administraron los pulsos eléctricos en cada lesión tumoral, recibiendo seis pulsos directos de corriente de $99 \mu \mathrm{s}$; hubo buena tolerancia al procedimiento (FIGURA 4).

El paciente presentó contracciones musculares con leve molestia al momento del procedimiento y, después, eritema en el área tratada con formación de ampolla en una de las lesiones; no presentó síntomas sistémicos. En el control a las dos semanas se evidenció ausencia de sangrado del tumor y disminución del tamaño de las lesiones (FIGURA 5); a las cuatro semanas había ausencia de las lesiones tratadas y del sangrado activo (FIGURA 6), es decir, hubo remisión clínica completa (desaparición completa del tumor).

\section{Caso 2}

Se trata de una mujer de 74 años de edad con antecedentes de carcinoma escamocelular verrucoso en vulva, tratada previamente con cirugía, radioterapia y criocirugía, con recurrencia tumoral.

El caso fue discutido en el Comité de Tumores por la persistencia del tumor y se decidió aplicar electroquimioterapia. En el examen físico presentaba una lesión tumoral de 6,5 x 3,5 cm en el área vulvo-inguinal izquierda (FIGURA 7). En la ecografía se reportó un volumen tumoral de $7 \mathrm{~cm}^{3}$.

Se trató bajo anestesia general, con aplicación de cuatro unidades de bleomicina intratumoral y pulsos eléctricos a los diez minutos, según el protocolo, haciendo varias aplicaciones según el tamaño del tumor, sin complicaciones. 
Presentó contracciones musculares al momento de generar los pulsos eléctricos sobre el tumor y, posteriormente, eritema, edema, ampollas y dolor en el área tratada, sin síntomas sistémicos asociados. En el control a las cuatro semanas se evidenció el tumor de igual tamaño (FIGURA 8), sin cambios en la lesión.

\section{Discusión}

La electroquimioterapia es una técnica desarrollada para el manejo coadyuvante del cáncer, que combina la quimioterapia (administración por vía intravenosa o intratumoral de un agente citotóxico, generalmente bleomicina o sales de platino) con la electropermeabilización de la membrana celular, generada por la aplicación local de impulsos eléctricos cortos e intensos que modifican transitoriamente sus propiedades, lo que resulta en la permeabilización reversible y facilita el paso de moléculas normalmente no permeables a su interior ${ }^{2}$. Este procedimiento fue realizado con bleomicina intratumoral, como terapia coadyuvante en dos pacientes con cáncer de piel en Manizales que no habían mejorado con terapias convencionales.

Esta técnica conlleva un aumento de la efectividad antitumoral de los medicamentos, con incremento de su citotoxicidad intracelular y una reducción notable de los efectos colaterales sistémicos, lo cual se evidenció en este estudio. Los pulsos eléctricos son aplicados con electrodos sobre la piel; se utilizan 6 a 8 impulsos de $99 \mu \mathrm{s}$ y $1.300 \mathrm{v}$ por centímetro a la frecuencia de repetición de un $\mathrm{Hz}$, es decir, uno por segundo. El protocolo es tan rápido que, si es necesario, se hacen varias aplicaciones para cubrir aquellas lesiones cuyo diámetro mida más de un centímetro. Los electrodos pueden ser externos para impulsos eléctricos transcutáneos o, también, pueden ser invasivos a base de agujas que penetran los tumores ${ }^{12}$. En los casos discutidos, se realizó el procedimiento utilizando los dos tipos de electrodos según la localización de las lesiones. Las lesiones profundas fueron manejadas con el electrodo de agujas y las lesiones superficiales con electrodos externos, con respuesta clínica similar en ellas.

Según los estudios disponibles, es una técnica segura y bien tolerada por los pacientes, con efectos secundarios leves: eritema, edema, contracción muscular producida en el momento de aplicación del impulso que desaparece inmediatamente después, quemaduras, ampollas, ulceración, dolor, necrosis superficial, prurito local y, excepcionalmente, náuseas ${ }^{13}$. Lo anterior fue corroborado en los casos tratados en el presente estudio, que presentaron contracciones musculares relacionadas con las descargas eléctricas, eritema, edema, ampollas y dolor después de la aplicación de la técnica, sin complicaciones ni aparición de síntomas sistémicos.

Las contraindicaciones reportadas hasta el momento son el uso de marcapasos cardíaco, hipersensibilidad a alguno de los medicamentos usados en el tratamiento y presencia de gases o líquidos inflamables ${ }^{14}$. Los dos pacientes manejados en este estudio, no presentaban contraindicaciones para la terapia.

En diversas series de casos se describe la tecnología como efectiva para ciertos tumores usando como medidas de resultado: sin respuesta, respuesta parcial (reducción de $50 \%$ ) y respuesta completa (desaparición completa del tumor) ${ }^{15-17}$, basados en estas experiencias, se decidió graduar la respuesta clínica en baja, intermedia y completa, modificando para el estudio los porcentajes de mejoría de la lesión. Se han reportado remisiones parciales y completas con este tratamiento, con mínimos efectos secundarios ${ }^{18-23}$. Grupos de investigadores en el área, como el de Mir, et al., han demostrado la eficacia, seguridad y buena tolerancia de la electroquimioterapia, remisión clínica satisfactoria hasta en $85,3 \%$ del total de casos evaluados, utilizando bleomicina intravenosa o intralesional en tumores cutáneos y subcutáneos, como carcinoma basocelular, melanoma, adenocarcinoma y carcinoma escamocelular en cabeza y cuello ${ }^{24-28}$, en el presente estudio fue bien tolerado el procedimiento por los pacientes, con respuestas clínicas variables y solo efectos colaterales locales.

Se utilizó bleomicina intratumoral aplicada según el volumen del tumor, medido previamente por ecografía. En el caso 1, se observó mejoría clínica de las lesiones en el control mensual, demostrada por disminución objetiva de su tamaño hasta su desaparición, es decir, se obtuvo remisión completa sin complicaciones, lo cual está de acuerdo con lo descrito en la literatura científica. El caso 2 fue manejado siguiendo el protocolo establecido, con dosis de bleomicina intratumoral, pero en el control no se observó remisión clínica, demostrado por la falta de cambios en el tamaño de la lesión tratada. Consideramos que la pobre respuesta clínica en este caso puede obedecer al alto grado de diferenciación del tumor.

Otros autores, como Sersa, et al., han evaluado la respuesta clínica a la electroquimioterapia con cisplatino, en pacientes con melanoma, carcinoma escamocelular y carcinoma basocelular. En un ensayo clínico de cuatro pacientes con 30 nódulos y tumores de diferentes tamaños, describieron remisión clínica completa a las cuatro semanas en 19 nódulos manejados con electroquimioterapia ${ }^{29,30}$, en los dos casos en cuestión se utilizó bleomicina intratumoral, se carece de experiencia con cisplatino utilizado en esta terapia en nuestro medio.

También, se han descrito cambios en el flujo sanguíneo tumoral con este tratamiento, ya que posee 
efectos vasculares que acentúan su acción antitumoral, siendo eficiente en casos de melanoma metastásico ulcerado que presentaban sangrado. Después del tratamiento intralesional con bleomicina y de la descarga de los pulsos eléctricos en todas las lesiones tratadas, se detuvo el sangrado de inmediato y no recurrió ${ }^{31,32}$. Este es un hallazgo demostrado en el presente estudio en el paciente con melanoma metastásico, en el cual se detuvo el sangrado activo que presentaba una de las lesiones después de recibir la electroquimioterapia, sin presentar recurrencia.

En otros estudios se reportan datos similares con buena respuesta clínica al tratamiento y escasos efectos secundarios, en pacientes con melanoma metastásico, constituyéndose así la electroquimioterapia, en una herramienta terapéutica útil en pacientes con recidivas o melanomas resistentes a otros tratamientos ${ }^{33-36}$.

\section{Conclusión}

La electroquimioterapia es una herramienta terapéutica novedosa y promisoria para el tratamiento coadyuvante o paliativo de pacientes con recidivas o cáncer de piel resistente a los tratamientos convencionales. Se utilizó en los dos pacientes de este estudio sin complicaciones ni efectos secundarios sistémicos, y con resultados alentadores en uno de ellos. Se requiere mayor experiencia para su recomendación y uso en nuestro país.

El desarrollo tecnológico y la implementación del protocolo de manejo clínico de esta investigación, abren nuevos caminos para generar experiencias locales y nuevos conocimientos en una técnica que, hasta donde se sabe, es la primera vez que se utiliza en el país.

\section{Agradecimiento}

Agradecemos a Consuelo Vélez Álvarez, asesora metodológica y enfermera epidemióloga, $\mathrm{PhD}$ en Salud Pública, de la Universidad de Caldas, y al ingeniero Jorge Hernán Estrada, profesor de Ingeniería de la Universidad Nacional de Colombia, sede Manizales.

\section{Referencias}

1. Sober A, Tsao H, Washington C. Cáncer de piel. En: Fauci AS, Braunwald E, Isselbacher KJ, Kasper DL, Hauser SL, Longo DL, et al. Harrison, Principios de Medicina Interna. Edición 17. Mexico DF: McGraw Hill interamericana; 2008. p. 547-54.

2. Nova J, Sánchez G, Porras L. Cáncer de piel: perfil epidemiológico de un centro de referencia en Colombia 2003-2005. Rev Salud Pública. 2007;9:595-601.

3. López G, Vanegas M, Escobar M. Registro poblacional (sic.) de cáncer Manizales - Caldas, resultados 2006. Quinquenio 20022006. Manizales: Universidad de Caldas; 2009.
4. Perfil epidemiológico de Manizales, sector salud. Manizales: Alcaldía de Manizales; 2008. Disponible en http://www.indicadoresmanizales.com. Consulta en año 2011

5. Santacoloma-Osorio G, Jaramillo-Ayerbe F. Comportamiento clínico, epidemiológico, e histológico del carcinoma basocelular en Caldas, Colombia. Med Cután Iber Lat Am. 1998;26:184-9.

6. Ospina JF, Jaramillo F. Comportamiento clínico y epidemiológico del carcinoma escamocelular cutáneo en el Hospital de Caldas de Manizales. Rev Soc Colomb Dermatol. 1999;7:70-3.

7. Villegas MP, Jaramillo F. Comportamiento clínico, epidemiológico e histológico del melanoma maligno en el Departamento de Caldas, Colombia. Rev Soc Colomb Dermatol. 1999;7:192-6.

8. Mesa J, Jaramillo-Ayerbe F, Benjumea M. Factores relacionados con la consulta tardía a dermatología por cáncer de piel. Prueba piloto. Departamento de Caldas. Hacia promoc salud. 2008;13:178-94.

9. Camué H, Segura D. La electroquimioterapia: una nueva alternativa terapéutica en la oncología. Rev Cubana Oncol. 2001;17:188-94.

10. Mir LM. Protocolo europeo de investigación en electroquimioterapia. Oncología. 2004;27:601-3.

11. Bergues L, Gómez L. La electroterapia: una alternativa terapéutica para el tratamiento de tumores. Rev Cubana Med [revista en Internet]. 2003;42. Disponible en: http://scielo.sld.cu/scielo.php. Consulta en año 2011

12. Mir LM, Belehradek M, Domenge C, Orlowski S, Poddevin B, Belehradek J, et al. Electrochemotherapy, a new antitumor treatment: First clinical trial. C R Acad Sci. 1991;313:613-8.

13. Sistema de Información de Tecnologías Sanitarias Nuevas y Emergentes. Ministerio de Sanidad y Consumo. Electroquimioterapia. Ficha técnica de nuevas tecnologías. Marzo de 2007. Disponible en: http://aunets.isciii.es. Consulta en año 2011.

14. Coringrato M, Jaled M, De Carli E, Cacabelos M. Radiofrecuencia ablativa en dermatología quirúrgica: una revisión. Dermatol Argent. 2008;14:228-33.

15. Australia and New Zealand Horizon Scanning Network. Electrochemotherapy. National Horizon Scanning. Unit Horizon scanning prioritizing. 2007;15:6. Disponible en: www.horizonscanning.gov.au. Consulta en año 2011.

16. Sersa G, Miklavcic D, Cemazar M, Rudolf Z, Pucihar G, Snoj M. Electrochemotherapy in treatment of tumours. Eur J Surg Oncol. 2008;34:232-40.

17. Muñoz V, Ortega G. Electrochemotherapy for treatment of skin and soft tissue tumours. Update and definition of its role in multimodal therapy. Clin Transl Oncol. 2011;13:18-24.

18. Heller R. Treatment of cutaneous nodules using electrochemotherapy. J Fla Med Assoc. 1995;82:147-50.

19. Glass LF, Pepine ML, Fenske NA, Jaroszeski M, Reintgen DS, Heller R. Bleomycin mediated electrochemotherapy of metastatic melanoma. Arch Dermatol. 1996;132:1353-7.

20. Glass LF, Fenske NA, Jaroszeski M, Perrott R, Harvey DT, Reintgen DS, et al. Bleomycin mediated electrochemotherapy of basal cell carcinoma. J Am Acad Dermatol. 1996;34:82-6.

21. Glass LF, Jaroszeski M, Gilbert R, Reintgen DS, Heller R. Intralesional bleomycin mediated electrochemotherapy in 20 patients with basal cell carcinoma. J Am Acad Dermatol. 1997;37:596-9. 
22. Byrne CM, Thompson JF. Role of electrochemotherapy in the treatment of metastatic melanoma and other metastatic and primary skin tumors. Expert Rev Anticancer Ther. 2006;6:671-8.

23. Giardino R, Fini M, Bonazzi V, Cadossi R, Nicolini A, Carpi A. Electrochemotherapy a novel approach to the treatment of metastatic nodules on the skin and subcutaneous tissues. Biomed Pharmacother. 2006;60:458-62.

24. Mir LM, Glass LF, Sersa G, Teissie J, Domenge C, Miklavcic D, et al. Effective treatment of cutaneous and subcutaneous malignant tumours by electrochemotherapy. Br J Cancer. 1998;77:2336-42.

25. Rodríguez-Cuevas S, Barroso-Bravo S, Almanza-Estrada J, Cristóbal-Martínez L, González-Rodríguez E. Electrochemotherapy in primary and metastatic skin tumors: Phase II trial using intralesional bleomycin. Arch Med Res. 2001;32:273-6.

26. Byrne CM, Thompson JF, Johnston H, Hersey P, Quinn MJ, Michael Hughes T, et al. Treatment of metastatic melanoma using electroporation therapy with bleomycin (electrochemotherapy). Melanoma Res. 2005;15:45-51.

27. Fantini F, Gualdi G, Cimitan A, Giannetti A. Metastatic basal cell carcinoma with squamous differentiation: Report of a case with response of cutaneous metastases to electrochemotherapy. Arch Dermatol. 2008;144:1186-8.

28. Landstrom FJ, Nilsson CO, Crafoord S, Reizenstein JA, Adamsson GB, Lofgren LA. Electroporation therapy of skin cancer in the head and neck area. Dermatol Surg. 2010;36:1245-50.
29. Sersa G, Stabuc B, Cemazar M, Jancar B, Miklavcic D, Rudolf Z. Electrochemotherapy with cisplatin: Potentiation of local cisplatin antitumour effectiveness by application of electric pulses in cancer patients. Eur J Cancer. 1998;34:1213-8.

30. Sersa G, Stabuc B, Cemazar M, Miklavcic D, Rudolf Z. Electrochemotherapy with cisplatin: Clinical experience in malignant melanoma patients. Clin Cancer Res. 2000;6:863-7.

31. Gehl J, Geertsen PF. Efficient palliation of haemorrhaging malignant melanoma skin metastases by electrochemotherapy. Melanoma Res. 2000;10:585-9.

32. Snoj M, Cemazar M, Srnovrsnik T, Kosir SP, Sersa G. Limb sparing treatment of bleeding melanoma recurrence by electrochemotherapy. Tumori. 2009;95:398-402.

33. Gaudy C, Richard MA, Folchetti G, Bonerandi JJ, Grob JJ. Randomized controlled study of electrochemotherapy in the local treatment of skin metastases of melanoma. J Cutan Med Surg. 2006;10:115-21.

34. Snoj M, Cemazar M, SlekovecKolar B, Sersa G. Effective treatment of multiple unresectable skin melanoma metastases by electrochemotherapy. Croat Med J. 2007;48:391-5.

35. Quaglino P, Mortera C, Osella-Abate S, Barberis M, Illengo M, Rissone $\mathrm{M}$, et al. Electrochemotherapy with intravenous bleomycin in the local treatment of skin melanoma metastases. Ann Surg Oncol. 2008;15:2215-22.

36. Kis E, Szegesdi I, OcsaiH, Gyulai R, Kemeny L, Olah J. Electrochemotherapy of melanoma cutaneous metastases. Orv Hetil. 2010;151:99-101. 